\title{
Literarische Anzeige.
}

Wathematische Annalen. Herausgegehen voa A.Clebsch und $C$. Neumann. Druck und Verlag von B.G. Teubner in Leipzig.

Bei dem stets wachsenden Umfange der mathematischen Disciplinen und bei der regen Production, welche dieselben seit Jahrzehnten in Deutschland hervorgerufen haben, wird schon seit längerer Zeit die Gründung eines neues mathematischen Journals von rein wissenschaftlicher Haltung vielseitig nicht nur fïr wïnschenswerth, sondern zur Erbillung eines dringenden Bedürfnisses selhst für nothwendig gehalten. In Uebereinstinmung mit dieser Ansicht fauden sich die beiden Unterzeichneten bereit, die Redaction einer solchen Zeitschrift zu äbernehmen, welche nnter dem Titel "Mathematische Annalen" in zwanglosen Heften erscheinen soll.

Dieselbe wird allen Originalarbeiten rein wissenschaftlichen Inhalts geöfnet sein, welche für das Gebiet der Mathematik selher oder für ihre wissenschaftlichen Anwendungen in irgend einer Weise förderlich sind. Dagegen geht die Absicht der Heransgeber weder auf Abfassung literarischer Berichte, noch auf Recensionen, um so weniger, als dieselben in einer in dem gleichen Verlage erscheinenden Zeitschrift bereits in ausgezeichneter Weise vertreten sind. Andererseits glauben die Unterzeichneten allerdings den Bedirfnissen des Publicums in so weit Rechnung tragen zu sollen, als sie den Wunsch ausdrücken, die ihnen ühersandten Aufsätze (durch geeignete Citate oder auch wohl durch kurze einleitende Worte) dem allgemeinen Verständniss möglichst vahe gebracht zu sehen.

Da Redaction und Verlagshandlung vor Allem die Dienste im Auge haben, welche sie der Wissenschaft zu leisten bestrebt sind, so wird es sowohl im Interesse der Autoren, als im Interesse des Publicums ganz vorzugsweise ihre Sorge sein, alle ihnen etwa anzuvertrauenden Arbeiten möglichst schoell zum Druck zu befördern. Um eine schnellere Publication zu ermöglichen, wird daher auch von einem hestimmten Maass für die Grösse eines Heftes Umgang genommen; dagegen soll jeder Band etwa die Stärke von 40 Bogen Lexicon-Octav erbalten und zu dem Preise von $5 \frac{\mathrm{l}}{3}$ Thlr. berechnet werden. Alle Buchhandlungen und Postanstalten nehmen Bestellungen an. Das erste Heft des 1. Bandes ist soeben erschienen und in allen Buchhandlungen zur Ansicht zu haben.

\section{Leiprig, im December 1868. Clebsch, Neumann.}

Inhalt des I. Heftes des 1. Bandes.

Ueher die Integration einer partiellen Differentialgleichung. Von Dr. H. Weber in Heidelberg.

Einige Eigenschaften einer gewissen Ordnung von Curven vierter Ordnung. Von J. Lüroth in Karlsruhe.

Note on the Solution of the Quartic Equation $\alpha U+6 \beta H=0$. By A. Cayley.

Ueber die Theorie der ternären cubischen Formen. Von A. Clebsch nnd P. Gordan in Giessen.

Ueber ternäre Formen dritten Grades. Von P. Gordan in Giessen.

Ueber die Doppeltangenten einer ebenen Curve vierten Grades. Von Dr. Geiser in Zürich.

Mittheilung über den III. Band von Gauss' Werken.

\section{I $\mathrm{n} \mathrm{h}$ a l $\mathrm{t}$.}

(Zu $\mathscr{Y}$ o 1734.) Astronomische Berechnungen und Beobachtungeu, angestellt auf der Kaiserl. Sternwarte zu Warschau. Mitgetheilt von Herrn Director Baronowski. Ueber die Bahnen der Cometen: IV. 1860, I. 1854 und IV. 1864. Von Herr Dr. J. Kowalczyk, Adj. der Warschauer Sternwarte. 81. -

Elemente des Planeten Arethusa (95). Von Herrn Dr. W. Schur. 91. -

Sonnen-Beobachtungen von 1868. Von Herrn Hofrath Schwabe. 93. -

Beobảchtungen auf der K. Sternwarte in Göttingen, mitgetheilt von Herrn Director, Prof. Klinkerfues. 95. -

(Zu $\mathscr{N}$ 1735-1736.) Schreiben des Herrn Barons Dembowski an den Herausgeber. 97. -

Schreiben des Herrn Dr. Kayser an den Herausgeber. 127. -

Auffindung der Antiope (90) in der dritten Erscheinung. Von Herrn H. Fogel. 127. -

(Zu $\mathscr{Y}$ 1737.) Schreiben des Herrn Professors Secchi an den Herausgeber. 129. -

Beobachtung der Sonnenfinsterniss am 29. August 1867. Mitgetheilt von Herrn L. Grosch. 137. -

Entdeckung eines neuen Planeten und Wiederauffindung der Sylvia, durch Herrn Norman R.Pogson, Director der Sternw. zu Madras. 139. Ueber die Wiederauffindung der Sylvia, von Dr. G. F. W. Peters. 141.-

Demande par rapport à la plauète Hécate. 141. - Literarische Anzeige. 143. -

Altona 1869. Januar 28. 